\section{Universal antidote for aptamers}

\section{By Kai-Jye Lou, Staff Writer}

Researchers at the Duke University School of Medicine have developed a strategy to reverse the activity of therapeutic aptamers in situations in which rapidly turning off the drug's effects is desirable, such as in cardiovascular surgery. ${ }^{1}$ The approach, which harnesses nucleic acid-binding polymers as the aptamer antidotes, could bolster the safety profiles of aptamers and give them a leg up over antibodies that go after the same targets.

Turning off the activity of an aptamer isn't exactly rocket science. The molecules are strings of nucleic acids, so rationally designed oligos that complement the aptamer sequence can modulate the aptamer's activity. Indeed, earlier work from Duke and Regado Biosciences Inc. had done just that. ${ }^{2,3}$

Regado's lead program is the REG1 Anticoagulation System, a two-component system composed of RB006, a single-stranded nucleic acid aptamer that binds to and inhibits Factor IXa, and RB007, a complementary nucleic acid that binds to and neutralizes RB006.

The catch is that designing an oligo to counteract an aptamer is not a cookie-cutter approach-a separate agent needs to be designed for each aptamer. This could be a costly endeavor, as each new control agent would also need to undergo testing along with its paired aptamer therapeutic.

To reduce the cost of developing control agents, the Duke researchers began a search for compounds that could reverse the effects of multiple aptamers in a sequence-independent manner, which led them to a class of polymers that target the backbone of the aptamers.

"The real eureka moment came when we realized that when we inject nucleic acid-based drugs into people, there normally aren't other free nucleic acids outside of cells in the bloodstream," said Bruce Sullenger, chief of the Division of Surgical Sciences at the Duke University School of Medicine and director of the Duke Translational Research Institute. "We found that compounds that recognized a component of the nucleic acid backbone, as opposed to a nucleic acid sequence, can be used to control the effect of aptamers."

Two of the polymers identified from a screen reversed the effects of eight different RNA aptamers. The researchers chose to focus on one of the polymers, $\beta$-cyclodextrin-containing polycation (CDP), as it had high binding affinity for the aptamers and is known to have low toxicity.

In pigs, $\mathrm{CDP}$ reversed the effects of an anticoagulant RNA aptamer within about five minutes.

Results were published in Nature Medicine. Sullenger was a senior author on the paper.

"The cyclodextrin approach is going to be a cheap and fast approach for reversing the effects of aptamer therapeutics," said John Rossi, chair of the Department of Molecular Biology at the Beckman Research Institute at City of Hope. "The development of a universal approach to control the activity of aptamer-based therapeutics makes the clinical use of these compounds more interesting."

"The ability to develop such agents without the need to develop more expensive customized antidote oligonucleotides would give aptamer-based therapeutics a decided advantage in terms of clinical development and safety," said Robert Schaub, SVP of R\&D at Archemix Corp.

"The work could address one of the key challenges that physicians face, particularly in the antithrombotic space. Once a physician deploys a therapeutic in a patient, he usually does not have the ability to recall the decision," said Christopher Rusconi, SVP of discovery and preclinical development and CSO of Regado. "Regado has invested a lot in the need to clinically control the effects of therapeutics, and the Nature Medicine paper further validates this as an important aspect in personalized medicine."

The aptamer antidote concept potentially could be applied to other types of nucleic acid-based therapeutics, he added.

In September, Regado began a Phase IIb trial evaluating REG1 as an anticoagulant therapy in acute coronary syndrome (ACS) patients undergoing cardiac catheterization. The biotech has an exclusive worldwide license from Archemix to discover and develop antidote-controlled aptamers for regulating fibrin deposition and platelet adhesion and aggregation under a 2003 deal.

Rusconi, Sullenger and former CEO Douglas Gooding, a venture partner at The Aurora Funds Inc., cofounded Regado in 2003.

Rusconi thinks a key question is how universal aptamer control agents would fit into the clinic. "How would these universal control agents be tested, and in what indications would their use be most relevant?" he asked.

Sullenger thinks the most immediate application of the universal antidotes is reversing the effects of antiplatelet and anticoagulant therapies.

"When a patient thinks they are having a heart attack or stroke, they are put on blood thinners to minimize the damage," he said. "However, if the patient needs surgery, doctors may not be able to operate until these drugs are cleared from the body. Bleeding is a big problem during 
surgical procedures and one clear application is to use these antidotes to reverse the blood-thinning effects of drugs given to patients prior to or during surgery if excessive bleeding does occur."

Heparin-protamine is the only anticoagulant-antidote pair that is routinely used in the clinic. But protamine is associated with side effects including reduced blood pressure, pulmonary hypertension, bradycardia and dyspnea.

Rusconi agreed that controlling the effects of blood thinners is important but said it remains unclear whether the universal control agents described in the paper will be able to tune the effects of aptamer therapeutics as opposed to just turning them off. "At least in the antithrombotic space, where use of control agents will be regular, physicians are not just looking for an on/off switch-they want control agents that can be used to tune or titrate the effects of the therapeutic," he told SciBX.

In a Phase Ic trial, Regado showed that physicians can use RB007 to create dose-dependent decreases in the activity of RB006. ${ }^{4}$

Rossi also noted that the universal control agent probably will not be able to target aptamers that get internalized by cells. He added that the Duke work only evaluated the control agent against RNA aptamers. "It would be important to evaluate the effect of these universal control agents against DNA aptamers as well," he told SciBX.

\section{Apt pupils}

Regardless of where CDP or other polymers will be applied, the researchers agreed that the availability of control agents-universal or specific-should encourage the development of more aptamer therapeutics.

"For potent aptamers targeting critical pathways or for those molecules designed for long action, the question of reversal is always raised," Schaub told SciBX. "The availability of a reversal agent could enhance the attractiveness of trials that include reversal agents for both the clinical investigator and the patient," he said.

Sullenger added that having the ability to control the effects of nucleic acid-based therapeutics also could help distinguish this drug class from antibody therapeutics.

Free antibodies are normally present in circulation, whereas free extracellular oligonucleotides are not. "It would be technically challenging and expensive to develop an antidote compound that could distinguish a therapeutic antibody from other endogenous antibodies in circulation," Sullenger noted.
Rossi thinks cyclodextrin-containing control agents are likely to have good safety profiles. He said similar cyclodextrincontaining polycation systems already are being evaluated in the clinic for the delivery of small interfering RNA and the chemotherapeutic camptothecin.

These include IT-101 and CALAA-01 from Calando Pharmaceuticals Inc., a subsidiary of Arrowhead Research Corp. IT-101, a conjugate of camptothecin and a cyclodextrin-based polymer, completed a Phase I trial in solid tumors in April. In June, Calando granted Cerulean Pharma Inc. exclusive worldwide rights to develop and commercialize IT-101.

CALAA-01 is an RNAi therapeutic that contains a cyclodextrin-based polymer and targets ribonucleotide reductase M2 (RRM2). The compound is in a Phase I trial to treat solid tumors.

Sullenger said the Duke group is now trying to develop universal aptamer control agents that are optimized and rationally designed. "We also want to see if we can do heart surgery in animal models and use these compounds to reverse the effects of anticoagulant aptamers," he said. He said he hopes to begin Phase I trials in one or two years and have them completed within two to three years.

Duke has filed multiple patent applications covering universal antidote technologies for nucleic acid-based aptamers. The work is available for licensing from the Duke University Office of Licensing \& Ventures. Regado licensed earlier Duke patents covering customized compounds that modulate the activity of nucleic acid aptamers.

Lou, K.-J. SciBX 2(40); doi:10.1038/scibx.2009.1494

Published online Oct. 15, 2009

\section{REFERENCES}

1. Oney, S. et al. Nat. Med.; published online Oct. 4, 2009;

doi:10.1038/nm.1990

Contact: Bruce A. Sullenger, Duke University School of Medicine, Durham, N.C.

e-mail: bruce.sullenger@duke.edu

2. Rusconi, C.P. et al. Nature 419, 90-94 (2002)

3. Rusconi, C.P. et al. Nat. Biotechnol. 22, 1423-1428 (2004)

4. Chan, M.Y. et al. J. Thromb. Haemost. 6, 789-796 (2008)

COMPANIES AND INSTITUTIONS MENTIONED

Arrowhead Research Corp. (NASDAQ:ARWR), Pasadena, Calif.

Archemix Corp., Cambridge, Mass.

The Aurora Funds Inc., Durham, N.C.

Beckman Research Institute at City of Hope, Duarte, Calif.

Calando Pharmaceuticals Inc., Pasadena, Calif.

Cerulean Pharma Inc., Cambridge, Mass.

Duke University School of Medicine, Durham, N.C.

Regado Biosciences Inc., Basking Ridge, N.J. 